\title{
Histological Patterns of Neurodegeneration of Frontal Cortex Neurons in Datura stramonium Treated Wistar Rats
}

\author{
Peter Etim Ekanem ${ }^{1}$, Regina Ekanem², Kidanemariam Gaim ${ }^{1}$ \\ ${ }^{1}$ Anatomy Unit, Biomedical Institute, College of Health Sciences, Mekelle University, Mekelle, Ethiopia \\ ${ }^{2}$ Public Health Department, Kampala International University, Kampala, Uganda \\ Email: "etimakpan@gmail.com, regina.ekanem@gmail.com, kidegaim@yahoo.com
}

Received 21 December 2015; accepted 1 February 2016; published 6 February 2016

Copyright (C) 2016 by authors and Scientific Research Publishing Inc.

This work is licensed under the Creative Commons Attribution International License (CC BY). http://creativecommons.org/licenses/by/4.0/

(c) (7) Open Access

\section{Abstract}

Aim: Datura stramonium (DS) is a known hallucinogen and depressant of the central nervous system, but it is commonly used in alcoholic beverages to increase intoxication. Pharmacological, physiological and ultra-structural studies have demonstrated the neurotoxicity of this drug inanimals and humans at high doses. The present study investigated the histological patterns of neurodegeneration of frontal cortex (FC) neurons in Wistar rats treated with high doses of DS seed extract. Materials and methods: Ethanolic extract of DS dried seeds was diluted in normal saline and administered to male and female Wistar rats weighing $200 \mathrm{~g}-250 \mathrm{~g}$. The animals were first placed in three groups which were further sub-divided into four sub-groups. The treated sub-groups received intraperitoneal administration (i.p.) of $750 \mathrm{mg} / \mathrm{kg}$ of diluted DS seed extract once daily in group 1, twice daily $(1500 \mathrm{mg} / \mathrm{kg} /$ day $)$ in group 2 and thrice daily $(2250 \mathrm{mg} / \mathrm{kg} /$ day $)$ in group 3 . The treatment was carried out for 4 weeks while the control groups received normal saline during the same period. The rats were euthanized and sections of the frontal cortices of the brain were histologically processed from all groups. Silver impregnation stain for degenerating axons and neurons was used to elucidate the pattern of degeneration induced by DS seed extract on the neurons of the FC. Results: The results of intraperitoneal administration of DS extract showed no changes in groups $1 \& 2$ treated rats while group 3 showed a significant pattern of histological changes like axonal atrophy, vacuolization and neuronal deaths in the frontal cortices neurons compared to the controls. Conclusion: DS may have a specific pattern of neurodegeneration at higher doses of administration. This could provide a useful model in understanding how DS intoxication can affect frontal cortex neurons with an implication of neurological disorders, mental diseases and behavioural deficits.

\footnotetext{
Corresponding author.
} 


\title{
Keywords
}

\author{
Pattern, Neurodegeneration, Datura stramonium, Frontal Cortex Neurons, Histological, Wistar \\ Rats
}

\section{Introduction}

Datura stramonium (DS), also referred to as Jimson weed, bears several other names based on different cultures because of its wide distribution and usage as a tropical shrub. It is a typical example of a hallucinogen with wide and varied uses. Some medicinal uses of the plant are its anti-inflammatory property found in all parts of the plant, stimulation of the central nervous system, respiratory decongestion, and treatment of dental and skin infections, alopecia and toothache [1]. It has been documented to be useful in the treatment of cough, chest pain, asthma, epilepsy, etc. [2]. In some cultures, it is used in alcoholic beverages to increase intoxication, and smoked in like manner as tobacco due to its psychoactive properties. It has also been reported to treat impotence and diarrhoea, kill parasites and act as a tipyretic for the control of fever [3].

Cases of poisoning have been reported after eating the berries [4]. Death may occur from heart failure after ingesting 125 seeds because the seeds contain the highest concentration of tropane alkaloids. The wide distribution, strong toxicity and potential for occurrence in foodstuffs are responsible for the numerous incidents of toxicity in humans [4]. Datura genus is distributed over tropical and warm temperate regions of the world. About ten species of Datura are found, of which Datura anoxia and D. stramonium are the most important drug plants. All parts of the plant are said to be toxic, most especially the seed. The toxins in Datura stramonium are tropane alkaloids. The major ones present include: hyoscine, atropine, scopolamine and hyoscyamine [5]. There possess strong anticholinergic properties, with scopolamine being a potent cholinergic blocking hallucinogen [5]. Over consumption of any part of the plant can, therefore, result in severe anti-cholinergic toxicity.

In addition, Datura stramonium has been documented to cause permanent short term memory loss, and is said to impair learning because it also contains a compound known as gamma-l-glutamyl-l-aspartate [6]. Moreover, due to its psychoactive properties, it is one of the major drugs implicated in the development of neurological disorders. These, according to WHO, are diseases of the central and peripheral nervous system, commonly referred to as mental disorders or "psychiatric illnesses" [7], and affect hundreds of millions of people worldwide [8].

Maibam et al. (2012) [9] found in their study that the administration of DS leaf extract at a certain concentration induced neurotoxicity in the cerebral cortex, as observed in electron photomicrographs which showed damaged and ruptured mitochondria as well as non-uniform broken nuclear membranes in the two experimental groups when compared with the control groups.

The human cerebral cortex contains network of neurons related to the initiation of movement, sensation from the body and the special sensory organs. It is the substrate for functions that include: comprehension, cognition, communication, reasoning, problem-solving, abstraction, imagination, planning and memory. The frontal cortex is the part of the cerebral cortex which extends from the central sulcus to the frontal pole. It is made up of 2-4 mm thick of highly convoluted sheath of grey mater with six layers which characterized the neocortex. The pyramidal cells are the main efferent neurons and their axons project to different areas of the central nervous system. Therefore, damage to these cells and their axons may lead to neurological disorder and behavioural deficits. It is one of the sites in the human brain vulnerable to the toxic effects of drugs. Studies in animals have revealed extensive axonal degeneration detectable in frontal cortices of rats following administration of d-amphetamine sulfate [10]. This indicates that several substances, such as drugs and tropical shrubs like DS, can affect the neurons of the frontal cortex and alter their morphology and roles.

Though pharmacological, physiological and ultra-structural studies of the frontal cortex have elucidated the possible neurotoxicity of DS seed, the effects of high doses of DS seed extract on the histological pattern of frontal cortex neurons and their degeneration have not been investigated. We, therefore, investigated the effects of high doses of DS seed extract on the neurons of the frontal cortices in male and female treated rats, and observed the pattern of degeneration to further understand the neurotoxic effect of this drug on the histology of the frontal cortex.

We hypothesize, in this work, that since the range of toxicity of the DS seed is highly variable and unpredictable (due to the varying concentrations of specific alkaloids with species, cultivation, environment, temperature, 
moisture and storage [11] [12]), excessive ingestion of high doses of DS seed extract may cause certain histological patterns of neuronal degeneration and cell loss.

\section{Materials and Methods}

\subsection{Plant Material Handling}

Datura stramonium (DS) seeds were harvested in Samaru, Zaria, Nigeria and identified in the herbarium of Biological Sciences, Ahmadu Bello University (A.B.U) and the Department of Pharmacognosy, Faculty of Pharmaceutical Sciences, A.B.U., Zaria. The seeds were extracted with $96.5 \%$ ethanol and phytochemical analysis conducted to identify the chemical composition of the extract. Dragendorff's reagent was used to confirm the presence of the major alkaloids and thin layer chromatography (TLC) was used to identify scopolamine, hyoscine, atropine and hyoscyamine as the major alkaloids.

\subsection{Animal Preparation for Experimentation}

Adult male and female Wistar rats, weighing $200 \mathrm{~g}$ - $250 \mathrm{~g}$ were used in this study. The animals were obtained from the animal houses of the Anatomy Department and the Veterinary Faculty, both of Ahmadu Bello University, (A.B.U), Zaria. The rules and regulations governing animal handling at A.B.U were strictly adhered to, and the experiment was conducted in accordance to the ethical committee guidelines.

A total of 48 experimental animals were randomly assigned to three groups of 16 animals per group. The groups were further sub-divided into four sub-groups, labelled A-D, of 4 animals per sub-group. Sub-groups A and $\mathrm{C}$ served as controls for both male and female rats in each group and received normal saline, while sub-groups $\mathrm{B}$ and $\mathrm{D}$ served as treatment sub-groups for both male and female rats respectively.

Bania et al. (2004) [13] proposed a pretreatment dose of $7.5 \mathrm{mg} / \mathrm{kg}$ atropine equivalent of DS seed extract as a protective agent in severe organophosphate toxicity. This was used as a reference for the calculation of the treatment doses. To induce intoxication, $7.5 \mathrm{mg} / \mathrm{kg}$ was increased tenfold since $\mathrm{LD}_{50}$ was found to be above $5000 \mathrm{mg} / \mathrm{kg}$. (The choice of this treatment was to establish the fact that high doses of this drug could lead to neuronal degeneration and the pattern of this degeneration in the neurons of FC). In the location where this study was carried out, many youth use this drug recreationally. Sub-groups B and D in each group were treated with different doses of DS extract. Group 1 received $750 \mathrm{mg} / \mathrm{kg}$, i.p. once daily, group 2 received $750 \mathrm{mg} / \mathrm{kg}$, i.p. twice daily (1500 mg/kg/day) and group 3 received $750 \mathrm{mg} / \mathrm{kg}$, i.p. thrice daily (2250 mg/kg/day) respectively.

This treatment was continued for 4 weeks, after which the animals were sacrificed by decapitation. Four weeks of treatment was employed to show that prolonged usage of this drug as in the case of addiction in high doses could produce histological changes in the morphology of the neurons of the FC, which also may contribute to many mental illnesses observed in the community in which the study was carried out.

The brains were dissected and the frontal cortices carefully removed from the rest of the brain, cut into pieces and fixed in $10 \%$ formalin for histological processing. The histological study was limited to the microscopic observations of the frontal cortices neurons in both male and female rats.

\subsection{Histology}

The tissues were processed in the Histology unit of the Anatomy Department at A.B.U., Zaria, Nigeria. The brain tissues were dehydrated in different grades of alcohol and cleared in xylene using an automatic processing machine (Shandon Southern Duplex Processor). The tissues were then infiltrated with paraffin wax and blocked in the coronal plane. Serial sections of the blocks were taken at $8 \mu \mathrm{m}$ with a (Leitz Wetzlar) microtome, mounted on glass slides and allowed to dry overnight. The staining technique employed was Glee's ammonia silver impregnation method for neurons and degenerating axons in paraffin sections [14]. Sections were then observed under a light microscope (SERICO, China) at low and high magnifications and micrographs taken with the help of video camera eyepiece (DCE-1 or DN-200M, China). Sections of the frontal cortices were observed in each of the male and female treated rats and compared to the controls.

\section{Results}

The histology of the frontal cortex neurons was examined under the microscope. Figure 1(a) and Figure 1(b) show the frontal cortices of the control male rats: (a) shows the general histological structure of the frontal cor- 
tex in low magnification, and in high magnification (b), neuronal cell bodies (yellow arrow) and axon (orange arrow) are seen. Figure 1(c), Figure 1(d) and Figure 1(e) show treated sub-groups of the three groups; Figure 1(c) and Figure 1(d) show that there were no significant changes in the cell bodies and axons while Figure 1(e) shows dead cells (red arrow), axonal atrophy (green arrow) and cytoplasm vacuolization (blue arrow).

Figure 2(a) and Figure 2(b) show the frontal cortices of the female control rats. Figure 2(a) shows the general section of the female frontal cortex under low magnification while Figure 2(b) is a higher magnification of the same section which shows the cell bodies (yellow arrow) and axon (orange arrow). Figure 2(c) is a high magnification of treated group 1 which shows that there were no significant changes in the cell bodies and axons, shown by the yellow arrow and orange arrow respectively, compared to the control. Figure 2(d) shows a large number of thick axons in the treated group 2, indicating no axonal atrophy or significant changes compared with the control. Figure 2(e) is a high magnification of treated group 3 and shows cell death (red arrow), axonal atrophy (green arrow) and cytoplasmic vacuolization (blue arrow).

The results showed that thrice daily intraperitoneal administration of DS seed extract ( $2250 \mathrm{mg} / \mathrm{kg} / \mathrm{day}$ ) for 4 weeks induced certain patterns of alterations in the histology of the frontal cortices' neurons of both male and female rats compared to the controls.
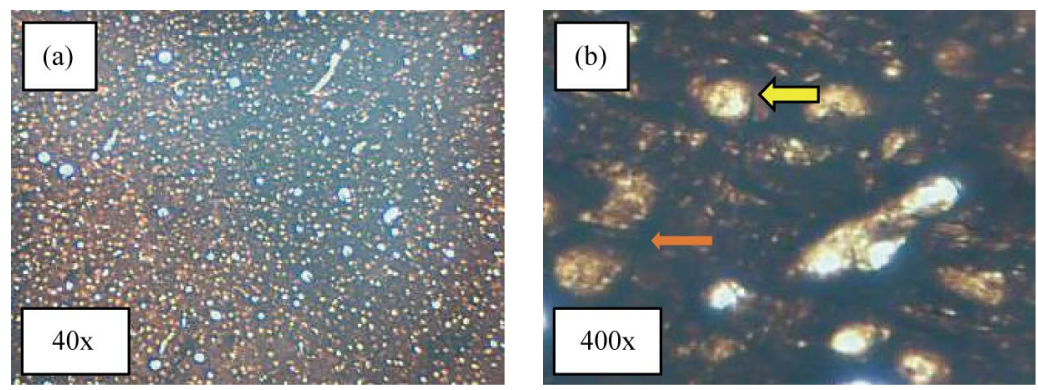

Male Untreated Control Rats
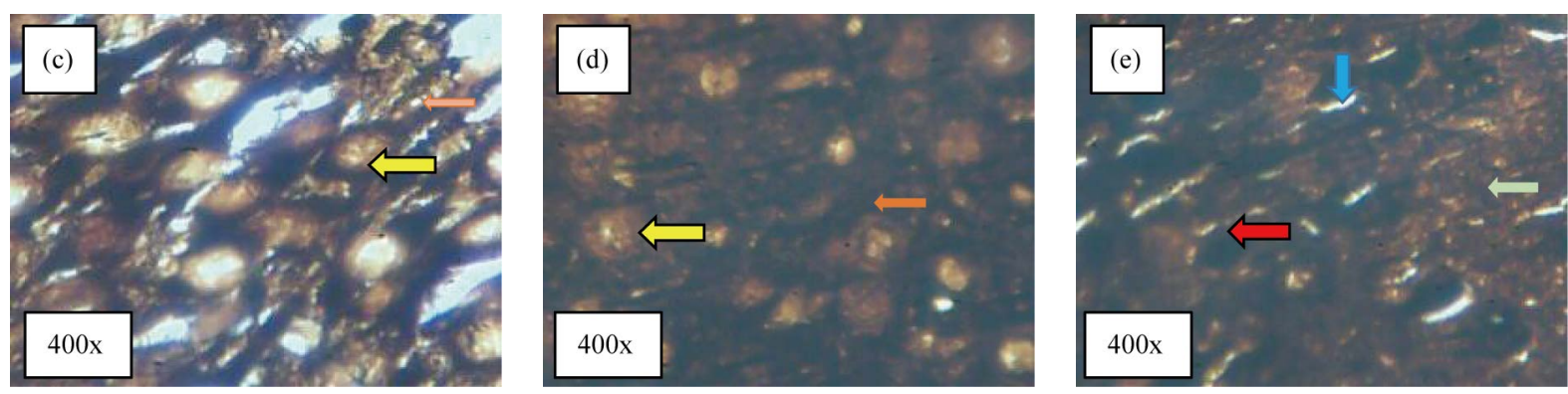

Male Treated Rat Groups

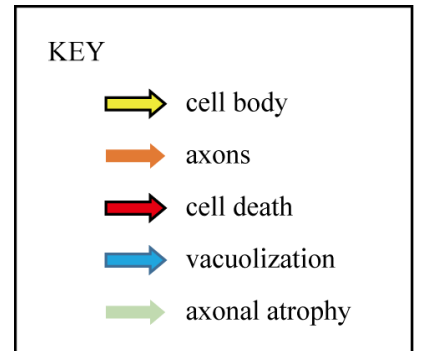

Figure 1. Effect of the DS extract treatment on the male Wistar rats' frontal cortices in Silver stain. (a) Coronal section of the control male rats' FC at low magnification (40x) showing general section of FC in male rat; (b) Coronal section of magnified section of the FC (mag.400x) showing prominent cell bodies of the neurons (yellow arrow) and thick axons (orange arrow); (c) Coronal section of FC in treated rats of group 1 FC (400x) showed prominent cell bodies (yellow arrow) and thick axons (orange arrow); (d) Coronal section of FC in treated rats group 2 with $1500 \mathrm{mg} / \mathrm{kg} / \mathrm{day}$ of DS seed extract at $750 \mathrm{mg} / \mathrm{kg}$ twice daily showed numerous thick axons (orange arrow) and cell bodies (yellow arrow) at magnification of 400x; (e) Coronal section of FC in the treated rat group 3 which received $2250 \mathrm{mg} / \mathrm{kg} /$ day at $750 \mathrm{mg} / \mathrm{kg}$ thrice daily showing cell death (red arrow), vacuolization (blue arrow) and axonal atrophy (green arrow) 400x. 


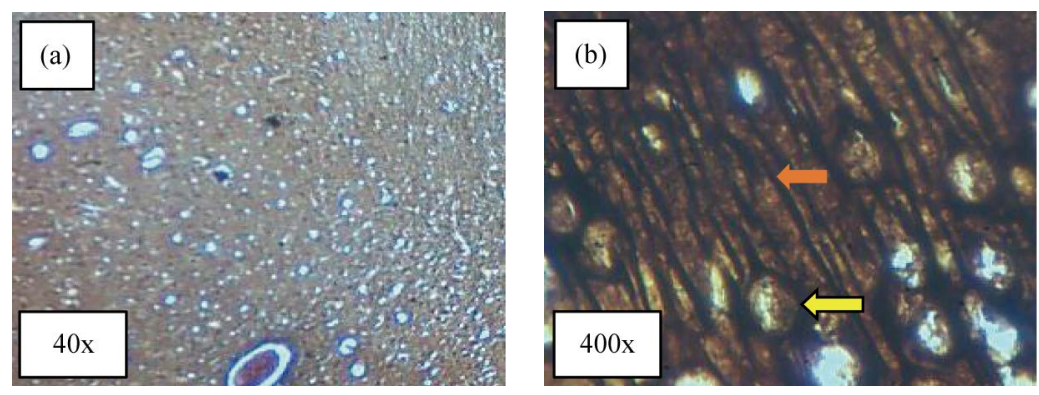

Female Untreated Control Group
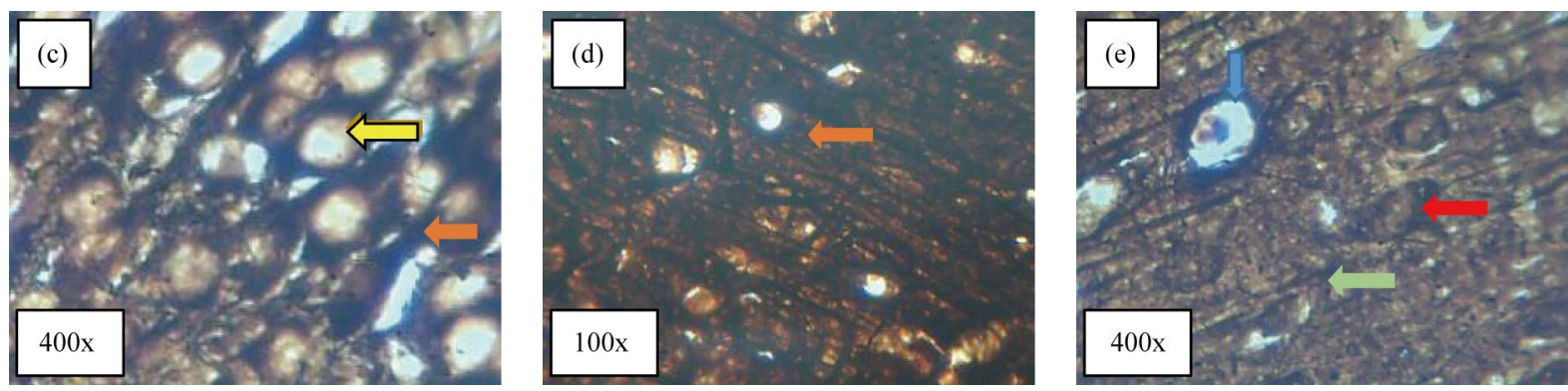

Female Treated Groups

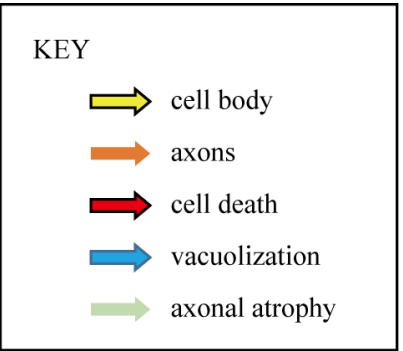

Figure 2. Effect of DS treatment on the female Wistar rats' frontal cortices in Silver stain. (a) Coronal section taken from control rats' FC at magnification of 40x. It showed general structure of female rats' FC; (b) Coronal section of FC at a magnification of 400x It showed prominent cell bodies (yellow arrow) and thick axons (orange arrow); (c) Coronal section of FC of group 1 treated rat's FC showing prominent cell bodies of the neuron (yellow arrow) and thick axons (orange arrow) as in control (400x); (d) Coronal section of FC of rats treated with $1500 \mathrm{mg} / \mathrm{kg} /$ day at $750 \mathrm{mg} / \mathrm{kg}$ twice daily of DS extract. It showed numerous and prominent ramifications of axons (orange arrow) 100x; (e) Coronal section of the FC of rats treated with $2250 \mathrm{mg} / \mathrm{kg} /$ day at $750 \mathrm{mg} / \mathrm{kg}$ thrice daily of DS extract. It showed cell death (red arrow), axonal atrophy (green arrow) and vacuolization (blue arrow) (400x).

\section{Discussion}

The treated sub-groups in group 1 which received $750 \mathrm{mg} / \mathrm{kg}$ of DS seed extract, i.p. once daily for 4 weeks and group 2 which received $750 \mathrm{mg} / \mathrm{kg}$ of DS seed extract, i.p. twice daily (1500 mg/kg/day) for 4 weeks did not show any significant changes in the histology of the neurons of the frontal cortices in both males and females, compared to their respective controls. Therefore, there were no definite patterns of neuro-degeneration recorded in the FC neurons of these groups compared to the controls. Also, there was no significant difference between male and female rats in these groups. This could imply that lower dosages may not produce a clear pattern of neurodegeneration in the frontal cortex neurons.

This result confirms the work of [15] carried out to study the effects of acute, subacute and chronic administration of alkaloids (atropine and scopolamine), the main constituents of the active component of $D$. stramonium. After acute i.p. administration of $100 \mathrm{mg} / \mathrm{kg}$ of total alkaloids in the seeds of $D$. stramonium, there were no remarkable changes in general appearance and no deaths occurred in any experimental group. Bouzidi et al. (2011) [16] conducted a chronic study using synthetic alkaloids administered i.p. at daily doses of $4.2 \mathrm{mg} / \mathrm{kg}$ of atropine and $1.6 \mathrm{mg} / \mathrm{kg}$ of scopolamine. These did not produce death. However, diarrhoea and hypoactivity were ob- 
served. The relative weight of the liver was significantly less than that of the control group. This shows that doses less than $2250 \mathrm{mg} / \mathrm{kg}$ of DS seed extract may not induce any significant changes in the histology of the frontal cortex neurons.

At such doses stated above, DS may actually have some benefits in treating certain diseases as documented in several literatures. Bania et al. (2004) [13] proposed a pretreatment dose of $7.5 \mathrm{mg} / \mathrm{kg}$ atropine equivalent of DS seed extract as a protective agent in severe organophosphate toxicity. Ardila and Moreno, (1991) [17] documented the medicinal uses of DS at low doses to include induction of mydriasis (pupillary dilation) and cycloplegia (paralysis of the eye focusing muscle) when used as eye drops. It is also used primarily in the treatment of eye disorders such as uveitis, iritis and iridocyclitis [17]. In dosage form, hyoscine, one of its alkaloid exists as tablets, drops, elixir, and injection and is used for the treatment of bladder spasms, peptic ulcer disease, diverticulitis, colic, irritable bowel syndrome, cystitis and pancreatitis [18].

On the other hand, treatment group 3 showed extensive atrophy of axons, vacuolization and cell death in both the male and female treated rats [750 mg/kg of DS seed extract, i.p. thrice daily (2250 mg/kg/day) for 4 weeks]. The patterns of these degenerative signs were the same in both male and female treated rats.These events could actually contribute to the clinical signs of excessive ingestion of DS seeds documented by [18], which include: headache, nausea, vomiting, blurred vision, dilated pupils, hot dry skin, dizziness, dryness of the mouth, difficulty in swallowing and CNS stimulation. In a study conducted by [9], they showed that administration of the DS plant extract at concentrations of $20 \mathrm{mg} / \mathrm{kg}$ and $40 \mathrm{mg} / \mathrm{kg}$ were found to induce neurotoxicity in the cerebral cortex, including the frontal cortex, as observed in the electron photomicrographs which showed damaged and ruptured mitochondria and non-uniform broken nuclear membranes in the two experimental groups when compared with the control groups. They also confirm the fact that the neurotoxicity of DS extract in their study showed a dose dependent damage. Adekomi et al. (2011) [19] found from their studies that exposing Sprague Dawley rats to the smoke of DS leaves induced histological changes in certain organs of the body compared to the control rats. Bihaqi et al. (2009) [20] showed that exposure to AlCl3 causes histopathological lesions in the cerebral cortex, including neuronal degeneration such as cytoplasmic vacuolization, hemorrhage, precellularodema and gliosis. DS seed extract may act through one of its components to induce neuronal cytoplasmic vacuolization as seen in the ALCl3 exposure in both male and female rats.

Maryam et al. (2013) [21] showed in their work that Vinca alkaloid, which has medicinal value for the treatment of certain diseases including the treatment regimens for testicular carcinoma, was found to be neurotoxic at higher doses. The primary pathologic effect observed was related to axonal degeneration and a decrease in axonal transport. Fowler (2014) [22], using histological analysis combined with stereological technique, demonstrated that the prefrontal cortex (PFC) which is part of the FC is vulnerable to chronic, alcohol-induced oxidative stress and neuronal cell death. Excessive oxidative stress and subsequent DNA damage can be responsible for neuronal apoptosis and neuronal dysfunction associated with different neurological pathologies [23]-[25]. The tropane alkaloids present in DS, like Vinca alkaloid, may have exerted their influence on the FC neurons causing axonal degeneration and neuronal cell death in both male and female treated rats. This confirms the fact that DS, which is like alcohol, is a CNS depressant, may cause neuronal cell death and loss in the FC.

Yagihashi et al. (1990) [26] also showed that axonal atrophy was related to a proportional loss of axonal neurofilaments in diabetic nerves. They asserted that the universal reduction of axonal size in diabetic nerves may be accounted for by impaired supply of neurofilaments or reduced neurofilament synthesis. Such cytoskeletal defects may, in turn, lead to distal axonal degeneration or contribute to the susceptibility of neurons to various external noxi, including ischemia and hypoglycemia [26]. Axonal atrophy observed in the neurons of the FC in both sexes could have been caused by loss of neurofilaments or reduction in their cytoskeletons, as in diabetic nerves. Studies in animals have also revealed extensive axonal degeneration detectable in the frontal cortices of rats following administration of d-amphetamine sulfate [10]. Because of the many chemical compounds in the DS, one or more of these components could have acted to produce axonal degeneration found in the neurons of the FC seen in this work. This indicates that, drugs such as DS, might induce axonal atrophy as one of the degenerative changes in the neurons of the frontal cortex and alter their roles.

Forrester (2006) [27] conducted a clinical and behavioral study in Texas from 1998-2004 on DS. He examined the patterns of human exposures to DS intoxication. The most reported clinical effects and behavioral deficits were hallucinations and confusion. Both clinical and experimental studies support a role of frontal cortical involvement in neuropsychological deficits in alcoholics, particularly those with Korsakoff's psychosis [28]. These behavioral deficits include dysfunction in emotional control, problem solving ability, and attention. Elec- 
trophysiologic studies using electroencephalograms and event related potentials have suggested that alcoholics have difficulty differentiating relevant and irrelevant, easy and difficult, and familiar and unfamiliar stimuli [29].

DS, which is sometimes used to increase intoxication in beverages like alcohol may exert its effect in a similar way as alcohol when ingested excessively. This may lead to atrophy of axons, cytoplasmic vacuolization and neuronal cell death observed in the frontal cortex and associated with certain behavioral deficit found in our society

\section{Conclusion}

In conclusion, the ethanolic extract of DS seeds given in excessive dosages was found to cause definite patterns of histological changes in the neurons of frontal cortices of the Wistar rats, such as cytoplasmic vacuolization, neuronal cell death and axonal atrophy in both males and females. These changes show that DS seed extract may have a histological pattern of neurodegeneration in the neurons of the FC of both male and female treated rats.

\section{Acknowledgements}

The researchers wish to acknowledge the staff of the Histology Unit, Anatomy Department of Anatomy, Ahmadu Bello University, Zaria, in supporting and providing technical assistance for conducting this research work.

\section{Limitations}

Paraffin wax infiltration and embedding was employed in this work in the absence of other better embedding media like celloidin that could have provided a better resolution in neuropathology sections and micrographs.

\section{References}

[1] Priyanka, S., Anees, A.S., Jawa, D. and Vishal, S. (2012) Pharmacological Properties of Datura stramonium L. as a Potential Medicinal Tree: An Overview. Asian Pacific Journal of Tropical Biomedicine, 2, 1002-1008. http://dx.doi.org/10.1016/S2221-1691(13)60014-3

[2] Wagner, A.D., Daniel, L.S., Rotte, M., Koutstaal, W., Maril, A., Dale, A.M., Rosen, B.R. and Buckner, R.L. (1998) Building Memories: Remembering and Forgetting of Verbal Experiences as Predicted by Brain Activity. Science, 281, 1188-1191. http://dx.doi.org/10.1126/science.281.5380.1188

[3] Bonde, K. (1998) The Genus Datura: From Research to Powerful Hallucinogen. Ethnobotanical Leaflets, 1998, Article 10. http://opensiuc.lib.siu.edu/cgi/viewcontent.cgi?article=1308\&context=ebl

[4] Das, S., Puneet, K. and Basu, S.P. (2012) Phytoconstituents and Therapeutic Potentials of DaturaStramonium. Linn. Journal of Drug Delivery \& Therapeutics, 2, 4-7. http://www.jddtonline.info/index.php/jddt/article/viewFile/141/79

[5] Arnett, A.M. (1995) Jimson Weed (Daturastramonium) Poisoning. Clinical Toxicology Review, 18, 1-6. https://www.erowid.org/plants/datura/datura_info5.shtml

[6] Schmitz-Bourgeois, M., Amiri, R.I., Boulanger, J. and Ungerer, Y. (1988) Isolation and Structure of a Pseudo Peptide Gamma-L-glutamyl-L-arspartatic Acid from Datura stramonium that Impairs Learning Retention in Mice. Biochimie, 70, 1179-1184. http://dx.doi.org/10.1016/0300-9084(88)90183-6

[7] WHO (2014) What Are Neurological Disorders? Online Question and Answer, Updated February 2014. http://www.who.int/features/qa/55/en/

[8] WHO (2014) Mental Health Neurological Disorders: Public Health Challenges. The World Health Report 2014. http://www.who.int/mental_health/neurology/chapter_2_neuro_disorders_public_h_challenges.pdf?ua=1

[9] Maibam, R.D., Meenakshi, B., Paul, S.B. and Sharma G.D. (2012) Characterization of the Toxic Effects Induced by Datura Stramonium Leaveson Mice: A Behavioral, Biochemical and Ultrastructural Approach. Asian Journal of Pharmaceutical and Clinical Research, 5, 143-146. http://www.ajpcr.com/Vol5Suppl3/1188.pdf

[10] Ryan, L.J., Linder, J.C., Martone, M.E. and Groves, P.M. (1990) Histological and Ultrastructural Evidence that D-Amphetamine Causes Degeneration in Neostriatum and Frontal Cortex of Rats. Brain Research, 518, 67-77. http://dx.doi.org/10.1016/0006-8993(90)90955-B

[11] Goldfrank, L.R. (1994) Toxicological Emergencies. 5th Edition, Appleton \& Lange, Norwalk, CT, 103-109.

[12] Haddad, L.M. and Winchester, J.F. (1990) Clinical Management of Poisoning and Drug Overdose. W.B. Saunders Co., New York, 345-350. 
[13] Bania, T.C., Chu, J., Bailes, D. and O’Neill, M. (2004) Jimson Weed Extract as Protective Agent in Severe Organophosphate Toxicity. Academic Emergency Medicine, 11, 335-338. http://dx.doi.org/10.1197/j.aem.2003.12.002

[14] Lillie, R.D. (1965) Histopathologic Technic and Practical Histochemistry. McGraw Hill Book Company, New York, 616-617.

[15] Gidado, A., Zainab, A., Hadiza, M.U., Serah, D.P., Anas, H.Y. and Milala, M.A. (2007) Toxicity Studies of Ethanol Extract of the Leaves of Datura stramonium in Rats. African Journal of Biotechnology, 6, 1012-1015.

[16] Bouzidi, A., Mahdeb, N. and Kara, N. (2011) Toxicity Studies of Alkaloids of Seeds of Datura stramonium and Synthesis Alkaloids in Male Rats. Journal of Medicinal Plants Research, 5, 3421-3431.

[17] Ardila, A. and Moreno, C.B. (1991) Scopolamine Intoxication as a Model of Transient Global Amnesia. Brain and Cognition, 15, 236-245. http://dx.doi.org/10.1016/0278-2626(91)90028-7

[18] Parotta, J.A. (2001) Healing Plants of Peninsular India. CABI Publishing, Wallingford and New York, 917.

[19] Adekomi, D.A., Musa, A.A., Tijani, A.A., Adeniyi, T.D. and Usman, B. (2011) Exposure to Smoke Extract of Datura stramonium Leaf: Some of its Effects on the Heart, Liver, Lungs, Kidneys and Testes of Male Sprague Dawley Rats. Journal of Pharmacognosy and Phytotherapy, 3, 67-75. http://www.academicjournals.org/jpp

[20] Bihaqi, S.W., Sharma, M., Singh, A.P. and Tiwari, M. (2009) Neuroprotective Role of Convolvulus pluricaulis on Aluminium Induced Neurotoxicity in Rat Brain. Journal of Ethnopharmacology, 124, 409-415. http://dx.doi.org/10.1016/j.jep.2009.05.038

[21] Maryam, M., Rusea, G.C., Yong, S.Y. and Mohd, N. (2013) Vinca Alkaloids. International Journal of Preventive Medicine, 4, 1231-1235.

[22] Fowler, A.-K., Thompson, J., Chen, L., Dagda, M., Dertien, J. and Dossou, K.S.S. (2014) Differential Sensitivity of Prefrontal Cortex and Hippocampus to Alcohol-Induced Toxicity. PLoS ONE, 9, e106945. http://dx.doi.org/10.1371/journal.pone.0106945

[23] Jacintho, J.D. and Kovacic, P. (2003) Neurotransmission and Neurotoxicity by Nitric Oxide, Catecholamines, and Glutamate: Unifying Themes of Reactive Oxygen Species and Electron Transfer. Current Medicinal Chemistry, 10, 2693-2703. http://dx.doi.org/10.2174/0929867033456404

[24] Ramalingam, M. and Kim, S.J. (2012) Reactive Oxygen/Nitrogen Species and Their Functional Correlations in Neurodegenerative Diseases. Journal of Neural Transmission, 119, 891-910. http://dx.doi.org/10.1007/s00702-011-0758-7

[25] Choi, D.H., Cristovao, A.C., Guhathakurta, S., Lee, J., Joh, T.H., Beal, M.F. and Kim, Y.S. (2012) NADPH Oxidase 1-Mediated Oxidative Stress Leads to Dopamine Neuron Death in Parkinson's Disease. Antioxidants and Redox Signaling, 16, 1033-1045. http://dx.doi.org/10.1089/ars.2011.3960

[26] Yagihashi, S., Kamijo, M. and Wantabe, K. (1990) Reduced Myelinated Fiber Size Correlates with Loss of Axonal Neurofilaments in Peripheral Nerve of Chronically Streptozotocin Diabetic Rats. American Journal of Pathology, 136, 1365-1373.

http://www.researchgate.net/publication/21015483 Reduced myelinated fiber size correlates with loss of axonal neurofilaments_in_peripheral_nerve_of_chronically_streptozotocin_diabetic_rats

[27] Forrester, M.B. (2006) Jimson Weed (Datura stramonium) Exposures in Texas, 1998-2004. Journal of Toxicology, Environment and Health, 69, 1757-1762. http://dx.doi.org/10.1080/15287390600631284

[28] Oscar-Berman, M. and Hutner, N. (1993) Frontal Lobe Changes after Chronic Alcohol Ingestion. In: Hunt, W.A. and Nixon, S.J., Eds., Alcohol-Induced Brain Damage, NIAAA Research Monograph No. 22, NIH Pub. No. 93-3549, National Institute on Alcohol Abuse and Alcoholism, Rockville, 121-156.

[29] Porjesz, B. and Begleiter, H. (1993) Neurophysiological Factors Associated with Alcoholism. In: Hunt, W.A. and Nixon, S.J., Eds., Alcohol-Induced Brain Damage, NIAAA Research Monograph No. 22, NIH Pub. No. 93-3549, National Institute on Alcohol Abuse and Alcoholism, Rockville, 89-120. 